\title{
An asymptotic Result for the Path Partition Conjecture
}

\author{
Marietjie Frick* \\ University of South Africa \\ PO. Box 392 \\ UNISA 0003 South Africa
}

\author{
Ingo Schiermeyer ${ }^{\dagger}$ \\ Technische Universität \\ Bergakademie Freiberg \\ 09596 Freiberg, Germany
}

Submitted: Apr 14, 2004; Accepted: Sep 1, 2005; Published: Sep 29, 2005

\begin{abstract}
The detour order of a graph $G$, denoted by $\tau(G)$, is the order of a longest path in $G$. A partition of the vertex set of $G$ into two sets, $A$ and $B$, such that $\tau(\langle A\rangle) \leq a$ and $\tau(\langle B\rangle) \leq b$ is called an $(a, b)$-partition of $G$. If $G$ has an $(a, b)$-partition for every pair $(a, b)$ of positive integers such that $a+b=\tau(G)$, then we say that $G$ is $\tau$-partitionable. The Path Partition Conjecture (PPC), which was discussed by Lovász and Mihók in 1981 in Szeged, is that every graph is $\tau$-partitionable. It is known that a graph $G$ of order $n$ and detour order $\tau=n-p$ is $\tau$-partitionable if $p=0,1$. We show that this is also true for $p=2,3$, and for all $p \geq 4$ provided that $n \geq p(10 p-3)$.
\end{abstract}

\section{Introduction}

The vertex set and edge set of a graph $G$ is denoted by $V(G)$ and $E(G)$, respectively. The degree of a vertex $v$ in $G$ will be denoted by $d_{G}(v)$. If $H$ is a subgraph of $G$, the open $H$-neighbourhood of $v$ is the set $N_{H}(v)=\{u \in V(H)-v \mid u v \in E(G)\}$. If $S$ is a subset of $V(G)$, we write $N_{H}(S)=\bigcup_{v \in S} N_{H}(v)$. The subgraph of $G$ induced by $S$ is denoted by $\langle S\rangle$.

A longest path in a graph $G$ is called a detour of $G$. The number of vertices in a detour of $G$ is called the detour order of $G$ and denoted by $\tau(G)$. The number of vertices in a longest cycle of $G$ is called the circumference of $G$ and denoted by $c(G)$. A graph

${ }^{*}$ This material is based upon work supported by the National Research Foundation under Grant number 2053752.

${ }^{\dagger}$ Part of this research was done while the author was on sabbatical visiting UNISA. Financial support by UNISA is gratefully acknowledged. 
of order $n$ will be called hamiltonian or traceable, if $c(G)=n$ or $\tau(G)=n$, respectively. The vertex independence number of a graph $G$ is denoted by $\alpha(G)$.

A partition of the vertex set of $G$ into two sets, $A$ and $B$, such that $\tau(\langle A\rangle) \leq a$ and $\tau(\langle B\rangle) \leq b$ is called an $(a, b)$-partition of $G$. If $G$ has an $(a, b)$-partition for every pair $(a, b)$ of positive integers such that $a+b=\tau(G)$, then we say that $G$ is $\tau$-partitionable. The following conjecture is known as the Path Partition Conjecture (or the PPC, for short).

Conjecture 1 Every graph is $\tau$-partitionable.

The PPC was discussed by Lovász and Mihók in 1981 in Szeged and treated in the theses [10] and [15]. The PPC first appeared in the literature in 1983, in a paper by Laborde, Payan and Xuong [11]. Although that paper dealt mainly with directed graphs, they stated the PPC only for undirected graphs. In 1995 Bondy [2] stated a directed version of the PPC. In [3] the PPC is stated in the language of the theory of hereditary properties of graphs. It is also mentioned in [5]. Results on the PPC and its relationship with other conjectures appear in [4], [6], [7], [8] and [9] . A summary of the conjecture status is given in [7].

A subset $S$ of $V(G)$ is called a $P_{n}$-kernel of $G$ if $\tau(\langle S\rangle) \leq n-1$ and every vertex $v \in V(G)-S$ is adjacent to an end-vertex of a path of order $n-1$ in $\langle S\rangle$ (cf. [5] and [13]). If $\tau(G)=a+b$ and $G$ has a $P_{a+1}$-kernel $S$, then $(S, V(G)-S)$ is an $(a, b)$-partition of $G$. It is shown in [6] that every graph has a $P_{n}$-kernel for every $n \leq 7$, and in [14] it is shown that every graph has a $P_{8}$-kernel. These results imply that the PPC holds for $a \leq 7$. However, Aldred and Thomassen [1] have recently constructed a graph that has no $P_{364}$-kernel.

\section{Main Results}

In this section we state our two main theorems, together with the main lemmas and the partition strategy used in the proofs. The proofs are presented in Section 4.

\section{The Partition Strategy}

Let $G$ be a graph of order $n$ and detour order $\tau=n-p$. Our main strategy is to find a subset $A_{1} \subset V(G)$ such that $\left|A_{1}\right|=p$ and $\left|N_{G-A_{1}}\left(A_{1}\right)\right| \leq \frac{\tau+1}{2}$.

If $\tau=a+b ; 1 \leq a \leq b$, and we can find such a set $A_{1}$, then we choose $B$ to be a subset of $V(G)-A_{1}$, consisting of exactly $b$ vertices and containing $N_{G-A_{1}}\left(A_{1}\right)$ (since $b \geq \frac{\tau+1}{2}$, this is possible). Then we set $A_{2}=V(G)-A_{1}-B$ and put $A=A_{1} \cup A_{2}$. Since $\left|A_{2}\right|=n-p-b=a$, it follows that $\tau(A) \leq \max \{a, p\}$. Thus $(A, B)$ will be an $(a, b)$-partition if $a \geq p$.

Since we know that the PPC holds for $a \leq 7$, our partition strategy will yield all the necessary partitions if $\tau\left(A_{1}\right) \leq 8$.

The following two lemmas will enable us to find all the necessary partitions when $p=3$, by applying our partition strategy. 


\section{Lemma 2.1}

(a) Let $P$ be a longest path in a nontraceable graph $G$ and let $H=G-V(P)$. If $H$ consists of $k \geq 1$ components, then $\left|N_{P}(H)\right| \leq \frac{\tau(G)-1}{2}+\left(\begin{array}{c}k \\ 2\end{array}\right)$.

(b) Let $C$ be a longest cycle in a nonhamiltonian graph $G$ and $H=G-V(C)$. If $H$ consists of $k \geq 1$ components, then $\left|N_{C}(H)\right| \leq \frac{c(G)}{2}+\left(\begin{array}{c}k \\ 2\end{array}\right)$.

Lemma 2.2 Let $G$ be a graph of order $n$ and detour order $\tau=n-p$ with $p \geq 2$, and let $P$ be a detour of $G$ with vertices labeled $v_{1}, v_{2}, \ldots, v_{\tau}$ such that $d_{P}\left(v_{1}\right) \leq d_{P}\left(v_{\tau}\right)$. If $H=G-V(P)$ consists of $k \geq 2$ components $H_{1}, H_{2}, \ldots, H_{k}$, then $\mid N\left(v_{1}\right) \cup N_{P}\left(H_{i}\right) \cup$ $N_{P}\left(H_{j}\right) \mid \leq \frac{\tau+1}{2}$, for $1 \leq i<j \leq k$.

Our first theorem follows from Lemma 2.1(a) and Lemma 2.2.

Theorem 2.3 Let $G$ be a graph of order $n$ and detour order $\tau=n-p$, with $0 \leq p \leq 3$. Then $G$ is $\tau$-partitionable.

When $n \geq p(10 p-3)$ the next lemma allows us to apply our partition strategy when $p \geq 4$, thus yielding $(a, b)$-partitions when $a \leq p$.

Lemma 2.4 Let $G$ be a graph of order $n$ and detour order $\tau=n-p$, with $p \geq 4$. Let $P$ be a detour of $G$ and let $H=G-V(P)$. If $\left|N_{P}(H)\right|>\frac{\tau+1}{2}$ then there exists an independent set $Y \subset V(P)$ with $|Y|=p$ such that $\left|N_{P-Y}(Y)\right| \leq \frac{\tau-1}{2}$, provided $n \geq p(10 p-3)$.

The next lemma enables us to find $(a, b)$-partitions when $a>p$, provided $n \geq 4 p^{2}-$ $6 p-4$.

Lemma 2.5 Let $G$ be a graph of order $n$ and detour order $\tau=n-p$, with $p \geq 1$. Suppose $\tau=a+b ; 1 \leq a \leq b$. If $a \leq \alpha(G)-p$, then $G$ has an $(a, b)$-partition.

Our second theorem uses Lemmas 2.1, 2.4 and 2.5, together with Lemmas 3.3 and 3.4.

Theorem 2.6 Let $G$ be a graph of order $n$ and detour order $\tau=n-p$, with $p \geq 4$. Suppose $\tau=a+b ; 1 \leq a \leq b$. Then the following hold:

(a) If $a \geq p$, then $G$ has an $(a, b)$-partition, provided $n \geq p(10 p-3)$.

(b) If $a<p$, then $G$ has an $(a, b)$-partition, provided $n \geq 4 p^{2}-6 p-4$.

Since $p(10 p-3) \geq 4 p^{2}-6 p-4$ for all $p \geq 4$, we have

Corollary 2.7 Let $G$ be a graph of order $n$ and detour order $\tau=n-p$, with $p \geq 4$. Then $G$ is $\tau$-partitionable, provided $n \geq p(10 p-3)$. 


\section{Auxiliary Results}

If $P$ is a path in $G$ with a fixed orientation and $u, v \in V(P)$, then $v^{-}$and $v^{+}$denote the immediate predecessor and immediate successor of $v$ on $P$, respectively. We denote the segment of $P$ from $u$ to $v$ by $u \vec{P} v$ and the reverse segment from $v$ to $u$ by $v \overleftarrow{P} u$. We shall refer to the vertices in the segment $u \vec{P} v$ as the interval $[u, v]$.

Lemma 3.1 Let $G$ be a connected nontraceable graph with detour order $\tau$ and let $P$ be a detour of $G$, with vertices labelled $v_{1} \ldots v_{\tau}$. Let $H=G-V(P)$ and let $H_{1}, \ldots, H_{k}$ be the components of $H$. Then the following hold:

(a) If $u \in V(P)$, then $N_{H_{i}}(u) \cap N_{H_{i}}\left(u^{+}\right)=\emptyset$, for $i=1, \ldots, k$.

(b) If $\{u, v\} \subseteq N_{P}\left(H_{i}\right)$ for some $i$, then $\left\{u^{+}, v^{+}\right\} \nsubseteq N_{P}\left(H_{j}\right)$ for any $j$.

(c) If $u \in N_{P}\left(v_{1}\right)$, then $u^{-} \notin N_{P}\left(v_{\tau}\right)$.

(d) If $u \in N_{P}(H)$, then $u^{+} \notin N_{P}\left(v_{1}\right)$ and $u^{-} \notin N_{P}\left(v_{\tau}\right)$.

\section{Proof.}

(a) Suppose $u$ and $u^{+}$both have neighbours in some component $H_{i}$ of $H$. Then a path of order greater than $\tau$ is obtained from $P$ by replacing the edge $u u^{+}$with a $u-u^{+}$ path whose internal vertices are in $H_{i}$.

(b) Suppose, to the contrary, that $\left\{u^{+}, v^{+}\right\} \subseteq N_{P}\left(H_{j}\right)$ for some $j$. Then it follows from (a) that $i \neq j$. Let $Q$ be a path in $H_{i}$ from a neighbour of $u$ to a neighbour of $v$ and let $R$ be a path in $H_{j}$ from a neighbour of $u^{+}$to a neighbour of $v^{+}$. Then the path $v_{1} \vec{P} u \vec{Q} v \overleftarrow{P} u^{+} R v^{+} \vec{P} v_{\tau}$ is longer than $P$.

(c) If $u^{-} \in N\left(v_{\tau}\right)$, then $v_{1} \vec{P} u^{-1} v_{\tau} \overleftarrow{P} u v_{1}$ is a cycle of order $\tau$ in $G$. But then there is a path of order $\tau+1$ in $G$ consisting of this cycle together with a vertex in $N_{H}(P)$.

(d) Let $h$ be a neighbour of $u$ in $H$. If $u^{+} \in N\left(v_{1}\right)$, then the path $h u \overleftarrow{P} v_{1} u^{+} \vec{P} v_{\tau}$ is longer than $P$. Thus $u^{+} \notin N\left(v_{1}\right)$. Similarly, $u^{-} \notin N\left(v_{\tau}\right)$.

Lemma 3.2 Let $G$ be a nontraceable graph with detour order $\tau$ and let $P$ be a detour of $G$, with vertices labelled $v_{1} \ldots v_{\tau}$. Let $H_{k}$ be a component of $H=G-V(P)$ and denote the neighbours of $H_{k}$ on $P$ by $u_{1}, \ldots, u_{s}$, labelled according to the order in which they appear on $P$. Then:

(a) $N_{P}^{+}\left(H_{k}\right)=\left\{u_{1}^{+}, \ldots, u_{s}^{+}\right\}$is an independent set.

(b) Consider any pair $i, j$, with $1 \leq i<j \leq s$ and suppose $x \in N_{P}\left(u_{i}^{+}\right)$. Then:

(i) If $x \in\left[v_{1}, u_{i}\right]$ or $x \in\left[u_{j}^{+}, v_{\tau}\right]$, then $x^{+} \notin N_{P}\left(u_{j}^{+}\right)$.

(ii) If $x \in\left[u_{i}^{++}, u_{j}\right]$, then $x^{-} \notin N_{P}\left(u_{j}^{+}\right)$. 


\section{Proof.}

(a) Suppose two vertices, $u_{i}^{+}, u_{j}^{+} \in N_{P}^{+}\left(H_{k}\right)$ are adjacent to one another. Let $Q$ be a path in $H_{k}$ from a neighbour of $u_{i}$ to a neighbour of $u_{j}$. Then the path $v_{1} \vec{P} u_{i} Q u_{j} \overleftarrow{P} u_{i}^{+} u_{j}^{+} \vec{P} v_{\tau}$ is longer than $P$. This contradiction proves that $N_{P}^{+}\left(H_{1}\right)$ is an independent set.

(b) (i) Let $Q$ be a path in $H_{k}$ from a neighbour of $u_{i}$ to a neighbour of $u_{j}$. Suppose $x^{+} \in$ $N_{P}\left(u_{j}^{+}\right)$. If $x \in\left[v_{1}, u_{i}\right]$, then the path $v_{1} \vec{P} x u_{i}^{+} \vec{P} u_{j} \overleftarrow{Q} u_{i} \overleftarrow{P} x^{+} u_{j}^{+} \vec{P} v_{\tau}$ is longer than $P$. If $x \in\left[u_{j}^{+}, v_{\tau}\right]$, then the path $v_{1} \vec{P} u_{i} Q u_{j} \overleftarrow{P} u_{i}^{+} x \overleftarrow{P} u_{j}^{+} x^{+} \vec{P} v_{\tau}$ is longer than $P$

(ii) If $x \in\left[u_{i}^{++}, u_{j}\right]$ and $x^{-} \in N_{P}\left(u_{j}^{+}\right)$then $v_{1} \vec{P} u_{i} Q u_{j} \overleftarrow{P} x u_{i}^{+} \vec{P} x^{-} u_{j}^{+} \vec{P} v_{\tau}$ is a path with more vertices than $P$.

The following result is proved in [8]

Lemma 3.3 Let $G$ be a graph and $(a, b)$ any pair of positive integers such that $\tau(G)=$ $a+b$. If $c(G) \leq b+2$, then $G$ has an $(a, b)$-partition.

The following Lemma was proved in [7].

Lemma 3.4 Let $G$ be a graph with $\tau(G)=a+b ; 1 \leq a \leq b$. If $G$ has a cycle $C$ of order greater than $b$ such that $\left|N_{C}(G-V(C))\right| \leq b$, then $G$ has an $(a, b)$-partition.

Corollary 3.5 Let $C$ be a longest cycle in a graph $G$. If $\left|N_{C}(G-V(C))\right| \leq\left\lceil\frac{\tau(G)}{2}\right\rceil$, then $G$ is $\tau$-partitionable.

Corollary 3.6 Let $C$ be a longest cycle in a graph $G$. If $\tau(G) \leq c(G)+1$, then $G$ is $\tau$-partitionable.

Proof. Two consecutive vertices of $C$ cannot both have neighbours in $G-V(C)$, otherwise $G$ would have a path of order $c(G)+2$. Thus $\left|N_{C}(G-V(C))\right| \leq\left\lceil\frac{\tau(G)}{2}\right\rceil$ and hence $G$ is $\tau$-partitionable, by Corollary 3.5.

\section{Proofs of the Main Results}

\section{Proof of Lemma 2.1.}

(a) If $H$ is connected, then $\left|N_{P}(H)\right| \leq \frac{\tau(G)-1}{2}$. So let $k \geq 2$. Suppose for two components of $H$, say $H_{1}, H_{2}$, the neighbourhood of $H_{1} \cup H_{2}$ contains three pairs of consecutive vertices $\left\{u, u^{+}\right\},\left\{v, v^{+}\right\}$and $\left\{w, w^{+}\right\}$on $P$. Assume $u \in N_{P}\left(H_{1}\right)$. Then, by Lemma 3.1(a) and (b), we must have $\left\{u, v^{+}\right\} \subseteq N_{P}\left(H_{1}\right)$ and $\left\{u^{+}, v\right\} \subseteq N_{P}\left(H_{2}\right)$. Now, by Lemma 3.1(a), either we have $w \in N_{P}\left(H_{1}\right)$ and $w^{+} \in N_{P}\left(H_{2}\right)$, or we have 
$w \in N_{P}\left(H_{2}\right)$,and $w^{+} \in N_{P}\left(H_{1}\right)$. By Lemma 3.1(b) the first case cannot occur, since we cannot have $\{u, w\} \subseteq N_{P}\left(H_{1}\right)$ and $\left\{u^{+}, w^{+}\right\} \subseteq N_{P}\left(H_{2}\right)$. Also, the second case cannot occur, since we cannot have $\{v, w\} \subseteq N_{P}\left(H_{2}\right)$ and $\left\{v^{+}, w^{+}\right\} \subseteq N_{P}\left(H_{1}\right)$. This proves that each of the $\left(\begin{array}{l}k \\ 2\end{array}\right)$ pairs of components of $H$ has at most two pairs of consecutive vertices on $P$ in their neighbourhood union. Thus, for each neighbour of $H$ on $P$, the next vertex is a non-neighbour, except in at most $2\left(\begin{array}{l}k \\ 2\end{array}\right)$ cases.

Since $v_{1}, v_{\tau} \notin N_{P}(H)$ and $P$ has $\tau$ vertices, we conclude that

$$
1+2\left|N_{P}(H)\right|-2\left(\begin{array}{l}
k \\
2
\end{array}\right) \leq \tau(G),
$$

and hence $\left|N_{P}(H)\right| \leq \frac{\tau(G)-1}{2}+\left(\begin{array}{l}k \\ 2\end{array}\right)$.

(b) By the same arguments as above we conclude that

$$
2\left|N_{C}(H)\right|-2\left(\begin{array}{l}
k \\
2
\end{array}\right) \leq c(G),
$$

which gives $\left|N_{C}(H)\right| \leq \frac{c(G)}{2}+\left(\begin{array}{l}k \\ 2\end{array}\right)$.

Proof of Lemma 2.2. If $d_{P}\left(v_{1}\right)+d_{P}\left(v_{\tau}\right) \geq \tau$, then there is a cycle containing $v_{1}, v_{2}, \ldots, v_{\tau}$, by Ore's Lemma. Since some vertex of $H$ is adjacent to some vertex on this cycle, we would have a path of order at least $\tau+1$ in $G$, a contradiction. Hence we may assume that $d_{P}\left(v_{1}\right)+d_{P}\left(v_{\tau}\right) \leq \tau-1$.

We shall call the case where $d_{P}\left(v_{1}\right)+d_{P}\left(v_{\tau}\right)=\tau-1$ the saturated case.

Let $H_{i}$ and $H_{j}$ be two components of $H$. If $N_{P}\left(H_{i}\right) \cup N_{P}\left(H_{j}\right) \subseteq N\left(v_{1}\right) \cup N\left(v_{\tau}\right)$, then it follows from Lemma 3.1 that $\left|N_{P}\left(H_{i}\right) \cup N_{P}\left(H_{j}\right) \cup N\left(v_{1}\right)\right| \leq \frac{\tau-1}{2}$. We may therefore assume that $q \geq 1$ vertices in $N_{P}\left(H_{i}\right) \cup N_{P}\left(H_{j}\right)$ are not neighbours of $v_{1}$ or $v_{\tau}$.

Suppose $N_{P}\left(H_{i}\right) \cup N_{P}\left(H_{j}\right)$ has $d$ pairs of consecutive vertices. As shown in the proof of Lemma 2.1, $d=0,1$, or 2 .

We call an interval $I=\left[v_{r}, v_{s}\right]$ a $t$-hole if $t=s-r+1$ and no vertex in $I$ is in $N\left(v_{1}\right) \cup N\left(v_{\tau}\right)$ but $v_{r-1}, v_{s+1} \in N\left(v_{1}\right) \cup N\left(v_{\tau}\right)$. We now compare the number of neighbours that $H$ can have in the holes of $P$ with the value that $d_{I}\left(v_{1}\right)+d_{I}\left(v_{\tau}\right)$ would have had in the saturated case. We need to consider three types of $t$-holes:

T1: $v_{r-1} \in N\left(v_{\tau}\right), v_{s+1} \in N\left(v_{1}\right)$ :

Since $v_{r+1}, v_{s-1} \notin N_{P}(H)$, it follows that $\left|N_{I}\left(H_{i}\right) \cup N_{I}\left(H_{j}\right)\right| \leq \frac{t-1+d}{2}$. In the saturated case, $d_{I}\left(v_{1}\right)+d_{I}\left(v_{\tau}\right)$ would have been equal to $t-1$.

T2: $v_{r-1} \in N\left(v_{1}\right)-N\left(v_{\tau}\right), v_{s+1} \in N\left(v_{\tau}\right)-N\left(v_{1}\right)$ :

In this case $\left|N_{I}\left(H_{i}\right) \cup N_{I}\left(H_{j}\right)\right| \leq \frac{t+1+d}{2}$, and in the saturated case $d_{I}\left(v_{1}\right)+d_{I}\left(v_{\tau}\right)$ would have been equal to $t+1$.

T3: $v_{r-1}, v_{s+1} \in N\left(v_{1}\right), v_{r-1} \notin N\left(v_{\tau}\right)\left(v_{r-1}, v_{s+1} \in N\left(v_{\tau}\right), v_{s+1} \notin N\left(v_{1}\right)\right)$ :

Since $v_{r-1} \notin N\left(v_{\tau}\right)\left(v_{s+1}\right) \notin N\left(v_{1}\right)$, it follows that $\left|N_{I}\left(H_{i}\right) \cup N_{I}\left(H_{j}\right)\right| \leq \frac{t+d}{2}$. In the saturated case $d_{I}\left(v_{1}\right)+d_{I}\left(v_{\tau}\right)$ would have been equal to $t$. 
Thus, in each hole $I$, the value that $d_{I}\left(v_{1}\right)+d_{I}\left(v_{\tau}\right)$ would have had in the saturated case is greater than or equal to $2\left|N_{I}\left(H_{i}\right) \cup N_{I}\left(H_{j}\right)\right|-d$. Since $H_{i} \cup H_{j}$ has altogether $q$ neighbours in the holes of $P$, we have

$$
\begin{aligned}
d_{P}\left(v_{1}\right)+d_{P}\left(v_{\tau}\right) & \leq \tau-1-(2 q-d) \\
& \leq \tau+1-2 q, \text { since } d \leq 2 .
\end{aligned}
$$

Hence $d\left(v_{1}\right) \leq \frac{\tau+1}{2}-q$ and therefore

$$
\begin{aligned}
\left|N\left(v_{1}\right) \cup N_{P}\left(H_{i}\right) \cup N_{P}\left(H_{j}\right)\right| & \leq\left(\frac{\tau+1}{2}-q\right)+q \\
& =\frac{\tau+1}{2} .
\end{aligned}
$$

Proof of Theorem 2.3. If $p=0,1$, then $G$ is $\tau$-partitionable (cf. [4]).

Now suppose $p \geq 2$ and $P$ is a detour of $G$ with vertices labelled $v_{1}, \ldots, v_{\tau}$, with $d\left(v_{1}\right) \leq d\left(v_{\tau}\right)$. Put $H=G-V(P)$. Then $|V(H)|=p$.

If $H$ has at most two components, put $A_{1}=H$. Then it follows from Lemma 2.1 that $\left|N_{G-A_{1}}\left(A_{1}\right)\right| \leq \frac{\tau+1}{2}$, so we get all the necessary partitions by applying our Partition Strategy.

If $H$ has three components, $H_{1}, H_{2}, H_{3}$, put $A_{1}=H_{1} \cup H_{2} \cup\left\{v_{1}\right\}$. Then it follows from Lemma 2.2 that $\left|N_{G-A_{1}}\left(A_{1}\right)\right| \leq \frac{\tau+1}{2}$, so again we get all the necessary partitions by applying our Partition Strategy.

Proof of Lemma 2.4. Let $u \in V(H)$ be a vertex which has a maximum number of neighbours on $P$. Then

$$
\left|N_{P}(u)\right|>\frac{\tau+1}{2 p}=\frac{n-p+1}{2 p} \geq \frac{10 p^{2}-4 p+1}{2 p}>5 p-2 .
$$

By Lemma 3.1(a) no vertex in $N_{P}^{+}(u)$ is adjacent to $u$ and by Lemma 3.1(b) no two vertices in $N_{P}^{+}(u)$ have a common neighbour in $H$. Hence at most $p-1$ vertices of $N_{P}^{+}(u)$ have neighbours in $H$. Let

$$
W=\left\{w \in N_{P}^{+}(u): N_{H}(w)=\emptyset\right\} .
$$

Then

$$
|W| \geq\left|N_{P}(u)\right|-(p-1) \geq 4 p .
$$

Let the vertices of $W$ be $w_{1}, \ldots, w_{r}$, labelled according to the order in which they appear on $P$. By Lemma 3.2(a), $W$ is an independent set.

Now let $I$ be an interval on $P$ such that all the vertices of $I$ except the first one is in $N(W)$. From Lemma 3.2 we deduce the following:

(1) The set $N_{I}\left(w_{i}\right)$ consists of consecutive vertices. 
(2) $\left|N_{I}\left(w_{i}\right) \cap N_{I}\left(w_{j}\right)\right| \leq 1$, for $1 \leq i<j \leq r$.

(3) If $I \subseteq\left[v_{1}, w_{1}^{-}\right]$, then the $I$-neighbourhoods of the vertices of $W$ appear in the order $N_{I}\left(w_{r}\right), N_{I}\left(w_{r-1}\right), \ldots, N_{I}\left(w_{1}\right)$. Moreover, if $1 \leq i<j \leq r$ and $\left|N_{I}\left(w_{i}\right) \cap N\left(w_{k}\right)\right|=1$, then $N_{I}\left(w_{k}\right) \subseteq N_{I}\left(w_{i}\right) \cap N\left(w_{j}\right)$ for all $k$ such that $i \leq k \leq j$.

(4) If $I \subseteq V\left[w_{s}, w_{s+1}^{-}\right]$for some $s \in\{1, \ldots, r-1\}$, then the $I$-neighbourhoods of the vertices in $W$ appear in the order $N_{I}\left(w_{s}\right), N_{I}\left(w_{s-1}\right), \ldots, N_{I}\left(w_{1}\right), N_{I}\left(w_{r}\right), N_{I}\left(w_{r-1}\right), \ldots$, $N_{I}\left(w_{s+1}\right)$. Now suppose $1 \leq i<j \leq r$ and $\left|N_{I}\left(w_{i}\right) \cap N\left(w_{j}\right)\right|=1$. Then the following hold:

If $j \leq s$ or $i \geq s+1$, then $N_{I}\left(w_{k}\right) \subseteq N_{I}\left(w_{i}\right) \cap N\left(w_{j}\right)$ for all $k$ such that $i \leq k \leq j$.

If $i \leq s$ and $j \geq s+1$, then $N_{I}\left(w_{k}\right) \subseteq N_{I}\left(w_{i}\right) \cap N\left(w_{j}\right)$ for all $k$ such that $k \leq i$ or $k \geq j$.

Let $q=\left\lfloor\frac{|W|}{p}\right\rfloor$ and put

$$
W_{i}=\left\{w_{(i-1) p+1}, \ldots, w_{i p}\right\} \text { for } 1 \leq i \leq q .
$$

Then $\left|W_{i}\right|=p$, for $i=1, \ldots, q$.

We now partition $P-v_{\tau}$ into consecutive intervals $I_{1}, \ldots I_{r}$ such that the initial vertex of each of the intervals is not in $N_{P}(W)$, while all the others are. It now follows from the structure of the $I_{j}$-neighbourhoods of the vertices in $W$ (as explained in (1)-(4) above) that

$$
\sum_{i=1}^{q}\left|N_{I_{j}}\left(W_{i}\right)\right| \leq\left|I_{j}\right|-1+q \text { for } j=1, \ldots, r .
$$

If $\left|I_{j}\right| \geq 3$, then $\left|I_{j}\right|-1+q \leq \frac{\left|I_{j}\right| q}{2}$, since $q \geq 4$. Furthermore, for each $i \in\{1, \ldots, q\}$, we have $\left|N_{I_{j}}\left(W_{i}\right)\right|=0$ if $\left|I_{j}\right|=1$ and $\left|N_{I_{j}}\left(W_{i}\right)\right| \leq 1$ if $\left|I_{j}\right|=2$. Thus

$$
\sum_{i=1}^{q}\left|N_{I_{j}}\left(W_{i}\right)\right| \leq \frac{\left|I_{j}\right| q}{2} \text { for } j=1, \ldots, r .
$$

Hence

$$
\sum_{i=1}^{q} \mid N_{P}\left(W_{i}\right) \leq \sum_{j=1}^{r} \frac{\left|I_{j}\right| q}{2}=\frac{(\tau-1) q}{2}
$$

and hence

$$
\min _{1 \leq i \leq q}\left|N_{P}\left(W_{i}\right)\right| \leq \frac{\tau-1}{2} .
$$

Now let $Y$ be a subset $W_{i}$ achieving this minimum. Then $\left|N_{G-Y}(Y)\right|=\left|N_{P-Y}(Y)\right| \leq \frac{\tau-1}{2}$.

Proof of Lemma 2.5. Let $A \subset V(G)$ be an independent set with $|A|=\alpha(G)$ and set $B=V(G)-A$. Then $\tau(\langle A\rangle)=1 \leq a$ and $\tau(\langle B\rangle) \leq n-\alpha(G) \leq \tau+p-(a+p)=b$.

Proof of Theorem 2.6. Let $P$ be a detour of $G$ and $H=G-V(P)$. 
(a) If $\left|N_{P}(H)\right| \leq \frac{\tau+1}{2}$ then, since $\tau(H) \leq p \leq a$, we can apply the Partition Strategy with $A_{1}=\bar{V}(H)$.

If $\left|N_{P}(H)\right|>\frac{\tau+1}{2}$, then by Lemma 2.5 there exists an independent set $Y \subset V(P)$ such that $N(Y)=N_{P}(Y)$ and $\left|N_{P}(Y)-Y\right| \leq \frac{\tau-1}{2}$. Now we can apply the Partition Strategy with $A_{1}=Y$.

(b) We distinguish two cases.

Case 1: $c(G) \leq n-2 p+3$ :

In this case

$$
b=\tau-a=n-p-a \geq n-p-(p-1)=n-2 p+1 .
$$

Thus we have $b \geq c(G)-2$. Hence $G$ has an $(a, b)$-partition by Lemma 3.3.

Case 2: $n-2 p+3<c(G)$ :

If $\alpha(G) \geq 2 p-1$, then $G$ has an $(a, b)$-partition by Lemma 2.5, so we may assume that $\alpha(G) \leq 2 p-2$. Let $C$ be a longest cycle of $G$. Let $H=G-V(C)$ and suppose $H$ has $k$ components. Then $k \leq \alpha \leq 2 p-2$. Let $\left|N_{C}(H)\right|=t$. By Lemma 3.4 we may assume that $b \leq t-1$ and by Lemma $3.3, t \leq \frac{c(G)}{2}+\left(\begin{array}{l}k \\ 2\end{array}\right)$. Thus

$$
b \leq \frac{c(G)}{2}+\left(\begin{array}{c}
2 p-2 \\
2
\end{array}\right)-1
$$

By Corollary 3.6 we may assume that $\tau \geq c(G)+2$. Now

$$
b=\tau-a \geq c(G)+2-(p-1) .
$$

It follows that

$$
\begin{aligned}
c(G)-p+3 & \leq \frac{c(G)}{2}+\left(\begin{array}{c}
2 p-2 \\
2
\end{array}\right)-1 ; \\
\text { i.e. } \quad c(G) & \leq 4 p^{2}-8 p-2 .
\end{aligned}
$$

But by our assumption, $c(G) \geq n-2 p+3$; hence

$$
\begin{aligned}
n-2 p+3 & \leq 4 p^{2}-8 p-2, \\
\text { i.e. } \quad n & \leq 4 p^{2}-6 p-5,
\end{aligned}
$$

contradicting our assumption.

Acknowledgement: We thank the referee for some helpful comments. 


\section{References}

[1] R.E.L. Aldred and C. Thomassen, private communication.

[2] J.A. Bondy, Handbook of Combinatorics, eds. R.L. Graham, M. Grötschel, and L. Lovász, The MIT Press, Cambridge, MA, 1995, Vol I, p. 49.

[3] M. Borowiecki, I. Broere, M. Frick, P. Mihók and G. Semanis̃in, A survey of hereditary properties of graphs, Discussiones Mathematicae Graph Theory 17 (1997), 5-50.

[4] I. Broere, M. Dorfling, J. E. Dunbar and M. Frick, A Pathological Partition Problem, Discussiones Mathematicae Graph Theory 18 (1998), 113-125.

[5] I. Broere, P. Hajnal and P. Mihók, Partition problems and kernels of graphs, Discussiones Mathematicae Graph Theory 17 (1997), 311-313.

[6] J.E. Dunbar and M. Frick, Path kernels and partitions, JCMCC, 31 (1999), 137-149.

[7] J.E. Dunbar and M. Frick, The Path Partition Conjecture is true for Claw-free Graphs, submitted.

[8] J.E. Dunbar, M. Frick and F. Bullock, Path partitions and $P_{n}$-free sets, Discrete Math. 289, No.1-3, (2004) 145-155.

[9] M. Frick and F. Bullock, Detour chromatic numbers of graphs, Discuss. Math. Graph Theory 21 (2001), 283-291.

[10] P. Hajnal, Graph Partitions (in Hungarian), Thesis, supervised by L. Lovász, J.A. University, Szeged, 1984.

[11] J.M. Laborde, C. Payan and N.H. Xuong, Independent sets and longest directed paths in digraphs, in: Graphs and other combinatorial topics (Prague,1982), 173177 (Teubner-Texte Math., 59, 1983.)

[12] L. Lovász, On Decomposition of Graphs, Studia Sci. Math. Hungar. 1 (1996) $237-$ 238.

[13] P. Mihók, Problem 4, p. 86 in : M. Borowiecki and Z. Skupien (eds), Graphs, Hypergraphs and Matroids (Zielona Góra, 1985).

[14] L.S. Melnikov and I.V. Petrenko, On the path kernel partitions in undirected graphs, Diskretn. Anal. Issled. Oper. Series 1, Vol. 9, No. 2 (2002) 21-35.

[15] J. Vronka, Vertex sets of graphs with prescribed properties (in Slovak), Thesis, supervised by P. Mihók, P.J. Safárik University, Košice, 1986. 